\title{
Optimised robot-based system for the exploration of elastic joint properties
}

\section{Journal Article}

\section{Author(s):}

Frey, M.; Burgkart, R.; Regenfelder, F.; Riener, R.

Publication date:

2004-09

Permanent link:

https://doi.org/10.3929/ethz-b-000160339

Rights / license:

In Copyright - Non-Commercial Use Permitted

Originally published in:

Medical \& Biological Engineering \& Computing 42(5), https://doi.org/10.1007/BF02347550 


\title{
Optimised robot-based system for the exploration of elastic joint properties
}

\author{
M. Frey ${ }^{1}$ \\ R. Burgkart ${ }^{2}$ \\ F. Regenfelder ${ }^{2}$ \\ R. Riener ${ }^{3, *}$ \\ ${ }^{1}$ Institute of Automatic Control Engineering, Technische Universität München, Germany \\ ${ }^{2}$ Clinic for Orthopaedics \& Sport Orthopaedics, Technische Universität München, Germany \\ ${ }^{3}$ Automatic Control Laboratory, Swiss Federal Institute of Technology (ETH) Zurich, Switzerland
}

\begin{abstract}
Numerous publications provide measured biomechanical data relating to synovial joints. However, in general, they do not reflect the non-linear elastic joint properties in detail or do not consider all degrees of freedom (DOF), or the quantity of data is sparse. To perform more comprehensive, extended measurements of elastic joint properties, an optimised robot-based approach was developed. The basis was an industrial, high-precision robot that was capable of applying loads to the joint and measuring the joint displacement in 6 DOF. The system was equipped with novel, custom-made control hardware. In contrast to the commonly used sampling rates that are below $100 \mathrm{~Hz}$, a rate of $4 \mathrm{kHz}$ was realised for each DOF. This made it possible to implement advanced, highly dynamic, quasi-continuous closed-loop controllers. Thus oscillations of the robot were avoided, and measurements were speeded up. The stiffness of the entire system was greater than $44 \mathrm{kN} \mathrm{m}^{-1}$ and $22 \mathrm{Nm} \mathrm{deg}^{-1}$, and the maximum difference between two successive measurements was less than $0.5 \mathrm{deg}$. A sophisticated CT-based referencing routine facilitated the matching of kinematic data with the individual anatomy of the tested joint. The detailed detection of the elastic varus-valgus properties of a human knee joint is described, and the need for high spatial resolution is demonstrated.
\end{abstract}

Keywords-Biomechanics, Knee kinematics, Robotics, Joint stiffness, Elastic joint properties

Med. Biol. Eng. Comput., 2004, 42, 674-678

\section{Introduction}

ONE METHOD to determine the elastic properties of human joints as a function of displacement is to apply load while the resulting displacement is recorded. Several authors have measured such data in vivo (MOORE et al., 1976; RIENER and EDRICH, 1999; TORZILLI et al., 1981). The main problem has been the moving soft tissue between the fixing of the measurement apparatus and the bones, which made precise measurements difficult to obtain. Furthermore, measurements of both an intact and an injured knee joint is only possible by accident for a single subject, as it is not justifiable to cause injury to a healthy person for research reasons. Hence, many groups have used human cadaver knee joints for biomechanical data acquisition.

Early measurement systems used different devices to apply load in different degrees of freedom (DOF) in a hand-operated manner (MARKOLF et al., 1976) or with weights (BLANKEVOORT et al., 1988). The load was measured with strain gauges, and the position was measured with externally fixed potentiometers (MARKOLF et $a l ., 1976)$ or with a camera system (BLACHARSKI et al., 1975). In

Correspondence should be addressed to Martin Frey:

email: martin.frey@paralab.balgrist.ch

*Also with Spinal Cord Injury Center, University Hospital Balgrist, Zurich, Switzerland

Paper received 23 December 2003 and in final form 26 April 2004

MBEC online number: 20043913

(C) IFMBE: 2004 the meantime, more advanced, partly automated set-ups have been developed. Single DOF were driven by hydraulic (PIZIALI and RASTEGAR, 1977) or electric (BERNS et al., 1990; DURSELEN et al., 1995; FUKUBAYASHI et al., 1982) actuators. However, these systems were limited in flexibility or in the number of DOF that could be actuated or measured. Furthermore, it was hard to guarantee that there were no constrained loads applied to the joint.

FUЛE et al. (1993) and WoO et al. (2000) used an industrial robot* in combination with a universal force torque sensor (FTS) (FUJIE et al., 1995) to measure elastic joint properties. In the meantime, other working groups picked up this idea. NEMEC et al. (2000) used a Riko 106 robot to test the kinematics of synovial joints. HURSCHLER et al. (2001) used a KUKA KR 15 robot to simulate arm forces while testing the shoulder joint. These set-ups were fully automated and able to move a joint and measure the load in 6 DOF simultaneously. Unfortunately, even modern industrial robots do not have a powerful control unit. Thus the sampling rate is very low (e.g. Stäubli RX 90-B, year of manufacture 2001, maximum sampling rate $62.5 \mathrm{~Hz}$ ). As a result, oscillations occur during measurement of quite stiff joints, or if the measurements take a long time.

The aim of this work was to develop an optimised robot based system that would enable fast, accurate and comprehensive measurements of elastic joint properties. For this purpose, new control hardware and software was developed. No oscillations

*Unimate Puma Model 762 
should occur during the measurements, and various measurement protocols have been be realised.

\section{Material and methods}

\subsection{Set-up}

The key element of the experimental set-up was an industrial, high-precision robot $^{\dagger}$, which is known as orto Marquet's surgical robot 'Caspar'. It is able to apply static forces of up to $120 \mathrm{~N}$ and torques of up to $10 \mathrm{Nm}$. It is equipped with a pneumatic safety device and a 6 DOF force torque sensor**.

The adjacent bones of a human cadaver joint were osteotomised $20 \mathrm{~cm}$ above and below the knee joint line. They were embedded into aluminium tubes with methylmethacrylat, and each bone was additionally fixed with three screws. Plastic cladding enclosed the cadaver to avoid dehydration. The tube with the thigh was mounted on the robot base (RB) (Fig. 1). The shank was attached to a plate that could be fixed to or dismantled from the FTS within seconds by quick-release skewers. The position of the cadaver leg relative to the robot was chosen such that the measurements were not constrained by singular constellations, which occur if at least two robot joints are aligned. This would have resulted in a reduction in the number of DOF, thus restricting the robot's mobility. The ethics committee of the university approved the study using human cadavers.

\subsection{Control hardware}

The robot was equipped with a novel, real-time, Linux-based controller developed in our group (HoOGEN et al., 2002). It runs on a Pentium IV with $1.9 \mathrm{GHz}$. The interface to the robot was realised with adequate PCI cards for the FTS signal, for the signals of the robot joint encoders and for the output signals that were used to control the motor currents of the robot joints. Thus the sampling rate could be increased by a factor of 64 up to $4 \mathrm{kHz}$ for each channel. This enabled us to implement highly dynamic, quasi-continuous control strategies.

\subsection{Co-ordinate systems}

The world co-ordinate system (CS) $\Sigma_{0}$ (index 0 ) was located in the RB, and the end effector (EE) CS $\Sigma_{E E}$ (index $E E$ ) was locked at the end of the robot arm (Fig. 2). The shank CS $\Sigma_{S}$ and the thigh CS $\Sigma_{T}$ were fixed to the cadaver. They were matched in full extension $\left(\Sigma_{S}=\Sigma_{T}\right)$. The choice of the co-ordinate system was based on the definition of GROOD and SUNTAY (1983), recommended by the ISB (WU and CAVANAGH, 1995). Modifications were made to obtain an orthogonal CS, and the descriptions of the axes were changed.

\subsection{Referencing routine, displacement and load calculation}

For referencing, the thigh and the shank were fixed, at full extension, by an external clamp. The pose of the shank $\mathrm{CS}^{B} \boldsymbol{T}_{S}$ and of the thigh $\mathrm{CS}^{B} \boldsymbol{T}_{T}$ with respect to the CT base (index $B$ ) were estimated by an experienced orthopaedic surgeon in a segmented and $3 \mathrm{D}$ reconstructed CT scan of the cadaver joint. Three artificial landmarks at the tubes clearly defined the reference $\mathrm{CS}^{B} \boldsymbol{T}_{R}$ with respect to the CT base. They were also located by a measuring device that was mounted on the robot. This resulted in the pose of the reference CS with respect to the robot base ${ }^{0} \boldsymbol{T}_{R}$. Thus the poses of the thigh CS ${ }^{0} \boldsymbol{T}_{T}={ }^{0} \boldsymbol{T}_{R}{ }^{R} \boldsymbol{T}_{B} \cdot{ }^{B} \boldsymbol{T}_{T}={ }^{0} \boldsymbol{T}_{R}\left({ }^{B} \boldsymbol{T}_{R}\right){ }^{-1} \cdot{ }^{B} \boldsymbol{T}_{T}$ and of the initial shank $\operatorname{CS}{ }^{0} \boldsymbol{T}_{S}\left(t_{0}\right)={ }^{0} \boldsymbol{T}_{R} \cdot\left({ }^{B} \boldsymbol{T}_{R}\right){ }^{-1} \cdot{ }^{B} \boldsymbol{T}_{S}$ with respect to the $\mathrm{RB}$ were clearly determined.

\footnotetext{
${ }^{\dagger}$ Stäubli RX90-B, Stäubli AG, Switzerland

†IPR GmbH, Germany

**JR3 Inc., USA
}

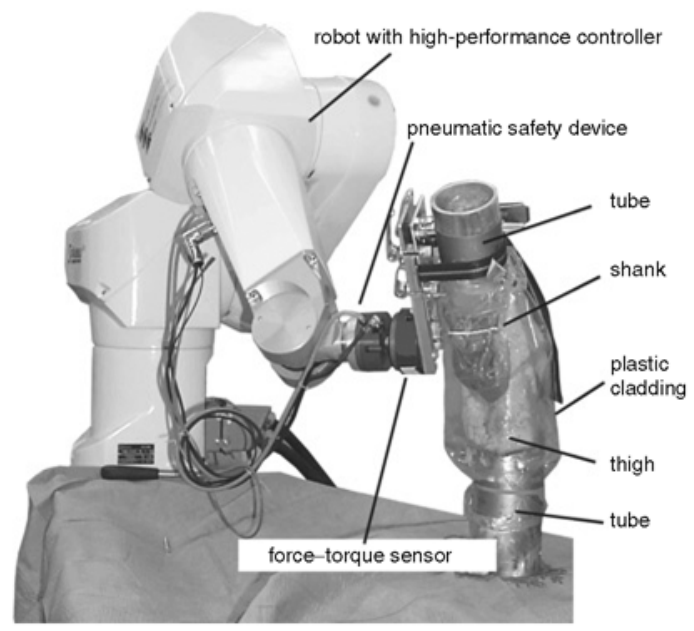

Fig. 1 Robot-based system

Now the robot was attached to the shank, and the initial EE position ${ }^{0} \boldsymbol{T}_{E E}\left(t_{0}\right)$ with respect to the RB was calculated from the robot kinematics. Then, the clamp fixing the aluminium tubes was dismantled. The pose of the shank ${ }^{0} \boldsymbol{T}_{S}$ with respect to the RB was given by ${ }^{0} \boldsymbol{T}_{S}={ }^{0} \boldsymbol{T}_{E E}{ }^{E E} \boldsymbol{T}_{0}\left(t_{0}\right) \cdot{ }^{0} \boldsymbol{T}_{S}\left(t_{0}\right)={ }^{0} \boldsymbol{T}_{E E}$. $\left({ }^{0} \boldsymbol{T}_{E E}\left(t_{0}\right)\right)^{-1} \cdot{ }^{0} \boldsymbol{T}_{S}\left(t_{0}\right)$ when the robot was moved. The actual displacement of the knee joint ${ }^{T} \boldsymbol{T}_{S}={ }^{T} \boldsymbol{T}_{0}{ }^{0} \boldsymbol{T}_{S}=\left({ }^{0} \boldsymbol{T}_{T}\right){ }^{-1} \cdot{ }^{0} \boldsymbol{T}_{S}$ was characterised by the pose of the shank relative to the thigh.

The displacement representation ${ }^{T} \boldsymbol{T}_{S}$ was transformed into a medical, non-orthogonal representation. The medical coordinates (MCs) were described by the FE rotation about, and the ML translation along, the $x$-axis of $\Sigma_{T}$. Then the VV rotation was performed about, and the AP translation was performed along, the actual flexed $y^{\prime}$-axis. Finally, the IE rotation and the PD translation were executed about and along the actual flexed and VV rotated $z^{\prime \prime}$-axis.

The FTS was located at the robot EE. According to the pose of the EE with respect to the thigh ${ }^{T} \boldsymbol{T}_{E E}={ }^{T} \boldsymbol{T}_{0} \cdot{ }^{0} \boldsymbol{T}_{E E}=\left({ }^{0} \boldsymbol{T}_{T}\right)^{-1}$. ${ }^{0} \boldsymbol{T}_{E E}$ the force $f_{E E}$ is transformed to $\Sigma_{T}$ by $f_{T}={ }^{T} \boldsymbol{R}_{E E} \cdot f_{E E}$ and the torque $t_{E E}$ is transformed by $t_{T}={ }^{T} \boldsymbol{R}_{E E} \cdot \boldsymbol{t}_{E E} \cdot\left(\boldsymbol{t}_{E E}+\right.$ $\left.\overrightarrow{\Sigma_{T} \Sigma_{E E}} \times \boldsymbol{f}_{E E}\right)$. The matrix ${ }^{T} \boldsymbol{R}_{E E}$ was the rotation matrix of ${ }^{T} \boldsymbol{T}_{E E}$, and $\overrightarrow{\Sigma_{T} \Sigma_{E E}}$ was the vector from the centre of $\Sigma_{T}$ to the centre of $\Sigma_{E E}$. Then, the forces and torques were transformed to MCs. Now, load and displacement were represented in MCs that were used for control.

\subsection{Control strategy}

It is possible to measure elastic joint properties in two principal ways: first, by moving the leg to a certain position and measuring the produced load, and, secondly, by applying a predefined load and measuring the caused displacement.

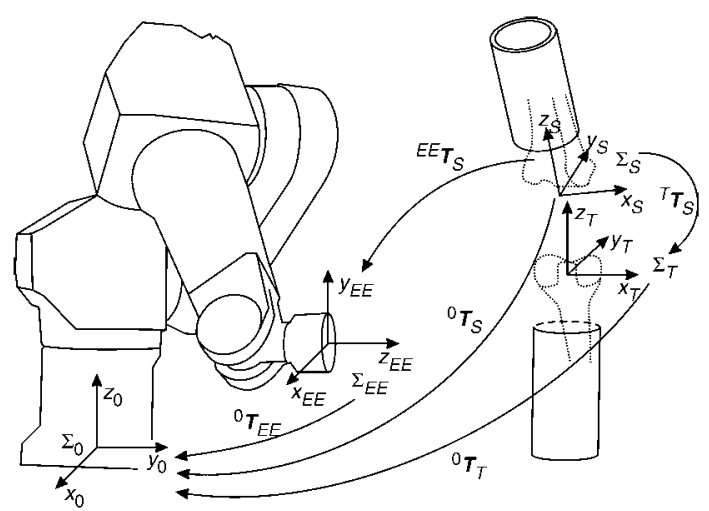

Fig. 2 Co-ordinate systems 


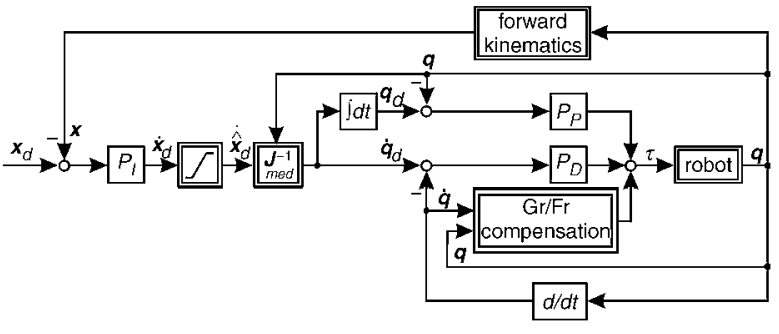

Fig. 3 Closed-loop position controller

The first method is optimum to measure with predefined position step width or regions with very low elasticity, e.g. the internal-external rotation DOF near zero load condition. The leg would rise to a large internal or external angle if a small, perhaps parasitic load were applied. Thus, it may be necessary to fix the joint to a certain position. Therefore a closed-loop position control was required. It was realised by a speed limited, closed-loop integral controller in MCs with a quite high gain $P_{I}$ and a subsidiary robot joint-based state-space position-velocity control (Fig. 3). The transformation between robot joint space $q$ and the MC $\boldsymbol{x}$ was made with the state-dependent, non-linear Jacobean matrix $J_{\text {med }}$ that was calculated from the robot kinematics and the transformations mentioned in Section 2.4.

The second method is used to avoid constrained loads in single DOF or to measure with a predefined load step width. This method requires a closed-loop force control. The actual load $f$ and the desired load $f_{d}$, both given in MCs, were compared. The difference was the input for an adaptive integral controller and the subsidiary robot joint-based position-velocity control mentioned above (Fig. 4). The gain of the outer control loop was on-line adapted as a function of the actual force. Additionally, there was a force feed-forward that was smoothed by a second-order filter (PT-2).

As there are large variations in the elastic properties among the single DOF of the knee joint, some DOF have to be positioncontrolled others and have to be load-controlled. This was realised by a hybrid closed-loop, load-position control (Fig. 5). It was a combination of the position controller in Fig. 3 and the force controller in Fig. 4. The choice of position or load control was made by the diagonal selection matrix $S\left(S_{i j}=0\right.$, if $\left.i \neq j\right)$. The signal of the position control loop was processed to the inner loop if $\boldsymbol{S}_{i i}=1$. Otherwise, if $\boldsymbol{S}_{i i}=0$, the output of the outer load control loop was passed to the inner loop. Thus each DOF could be either load or position controlled.

\subsection{Accuracy}

The relative position error in Cartesian space was specified to be lower than $0.02 \mathrm{~mm}$. The stiffness of the set-up, including mounting device and force sensor, was measured. Therefore an aluminium tube was fixed to the RB, and the robot was attached to the tube. Load was applied to the tube, and the displacement was recorded. The resulting transverse stiffness

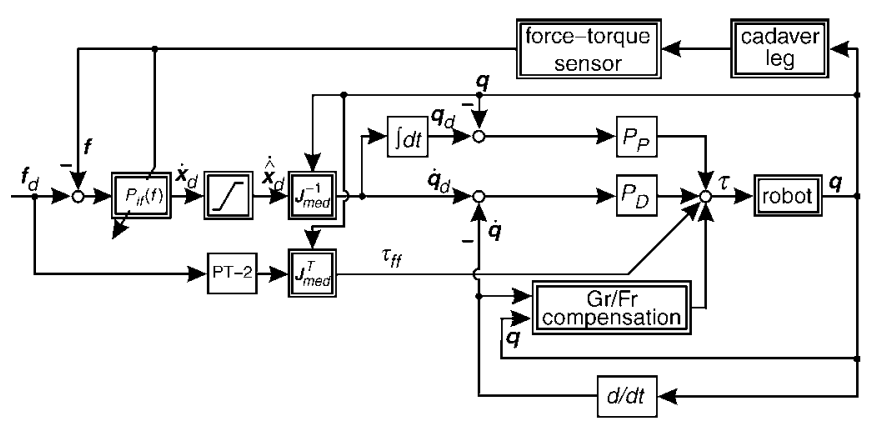

Fig. 4 Closed-loop load controller

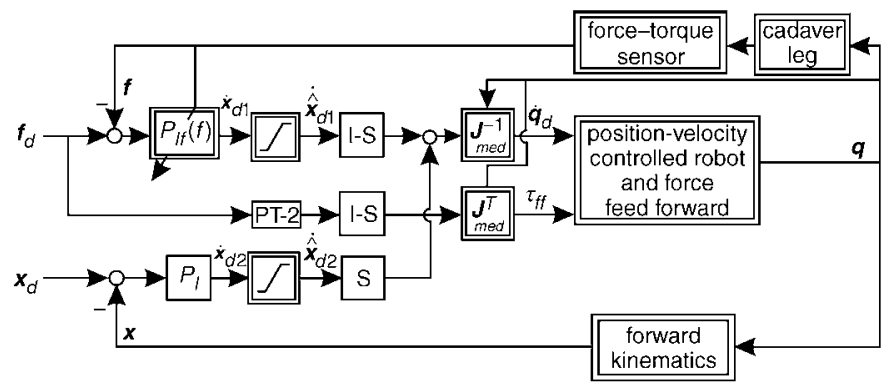

Fig. 5 Hybrid closed-loop load-position controller

was between $44 \mathrm{kN} \mathrm{m}^{-1}$ and $188 \mathrm{kN} \mathrm{m}^{-1}$, depending on the robot constellation. The rotary stiffness was higher than $22 \mathrm{Nm} \mathrm{deg}^{-1}$.

\subsection{Protocol}

As an example, the protocol of measuring the elastic varusvalgus properties as a function of the flexion angle is presented.

The flexion DOF was position controlled, and the varus DOF was load controlled. While one or the other was varied, the other DOF was fixed to a certain value. The fixed DOF could be the flexion angle (top of Fig. 6) or the varus moment (bottom of Fig. 6). Varus load varied between $-10 \mathrm{Nm}$ and $10 \mathrm{Nm}$, and the flexion angle varied between hyperextension $\left(-5^{\circ}\right)$ and full flexion $\left(120^{\circ}\right)$. All translatory DOF were zero force controlled to avoid reactive forces. The internal-external rotation DOF was fixed to the zero rotation angle that was recorded during initialisation of the cadaver.

The movements with fixed flexion DOF were comparable with the execution of a varus-valgus stress test (RITCHIE et al, 1994). The measurements resulted in a hysteresis curve for each flexion angle (Fig. 6c). The measurements with fixed valgus loads resulted in level curves of the elastic valgus properties (Fig. 6f). Fig. $6 f$ displays the flexion-dependent valgus displacement at a constant valgus load.

\section{Results}

After testing and optimising of the experimental set-up with five animal knee joints, the elastic characteristics of three fresh frozen human knee joints were analysed. Therefore individual properties of the intact, as well as the pathological, knee joint were determined in $6 \mathrm{DOF}$ and with high spatial resolution, to be applicable for the Munich knee joint simulator (RIENER et al., 2004; FREY et al., 2003a).

As an example, the detection of the passive elastic varusvalgus properties as a function of the knee flexion angle is described. Approximately $30 \mathrm{~min}$ were required to run the basic protocol. Both the displacement and load data were recorded in $6 \mathrm{DOF}$ at flexion angles of $-5^{\circ}, 0^{\circ}, 10^{\circ}, 20^{\circ}, 30^{\circ}$, $40^{\circ}, 60^{\circ}, 80^{\circ}, 100^{\circ}$ and $120^{\circ}$. At each flexion angle, the varus load was increased up to $10 \mathrm{Nm}$, then decreased to $-10 \mathrm{Nm}$ and again increased up to $0 \mathrm{Nm}$ in steps of $1 \mathrm{Nm}$. The setting of the protocol and two cycles of preconditioning at each flexion angle were also included within this time frame.

Regardless of the relative accuracy of the used robot, the point of interest is the repeatability of the measurements performed. To analyse this aspect, the protocol mentioned above was completed twice with varus load increments of $0.25 \mathrm{Nm}$ and $0.5 \mathrm{Nm}$ (Fig. 7). In the first measurement, the robot EE was mounted medially of the tibia, and, in the second measurement, it was mounted frontally. The maximum deflection between the two measurements was $0.25^{\circ}$ at the maximum load of $10 \mathrm{Nm}$. 

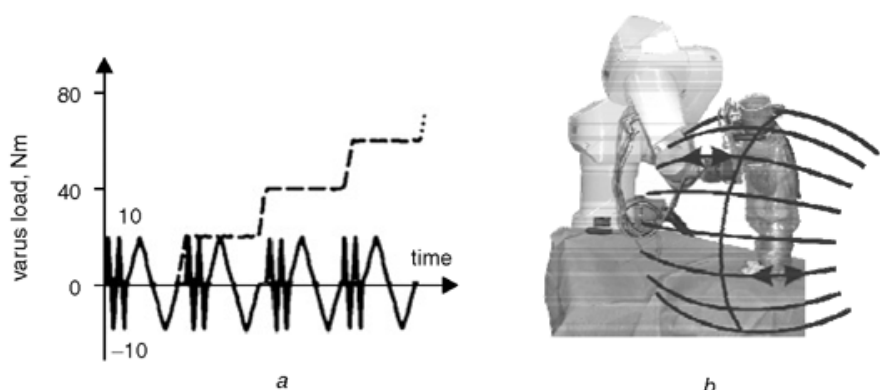

$b$

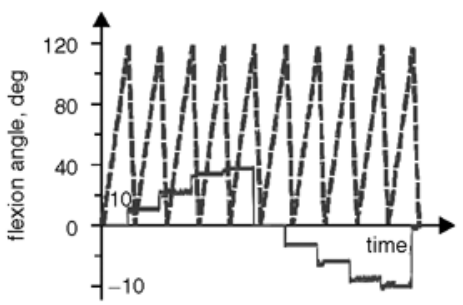

$d$

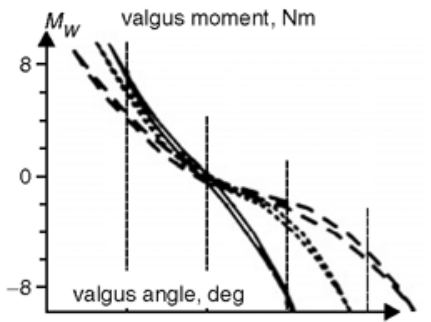

$c$

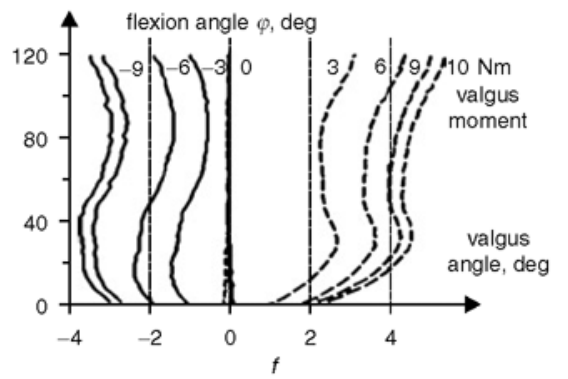

Fig. 6 (a), (d) Measurement protocols and (c), (f) resulting curves. Protocol with fixed flexion angle is displayed on top; protocol with fixed varus load is displayed at bottom. (a) (--) Flexion angle, deg; (-) valgus moment, Nm. (c) Flexion angle: (-) 0 deg; (--) 4 deg; (--) 100 deg;

This was in the same range as the error caused by the posedependent stiffness of the set-up.

For all cadaver legs, highly non-linear and non-symmetric characteristics of the elastic varus-valgus properties were measured. At flexion angles larger than $60^{\circ}$, there was a sharp bend close to zero load (Fig. $7 a$ ). A possible explanation is that the lateral femur condyle lifts off the lateral tibia plateau when varus load is applied, as the lateral collateral ligament is relaxed at $80^{\circ}$ of knee flexion (BRANTIGNAN and VOSHELL, 1941). During valgus load, the lateral femoral condyle is then in contact with the tibia plateau, with simultaneous tensioning of the medial collateral ligament. Thus the elastic stiffness increases with a high slope.

The protocol was processed again, but with a spatial resolution of $2 \mathrm{Nm}$. The measured points were interpolated with cubic splines. The resulting curve is almost symmetrical (Fig. $7 b$ ), as is reported by MARKOLF et al. (1976). Details such as the sharp bend in the varus-valgus characteristics are lost as a result of the low resolution.

\section{Discussion}

The following outstanding aspects of the presented robotbased set-up for the detection of passive elastic synovial joint properties should be emphasised.
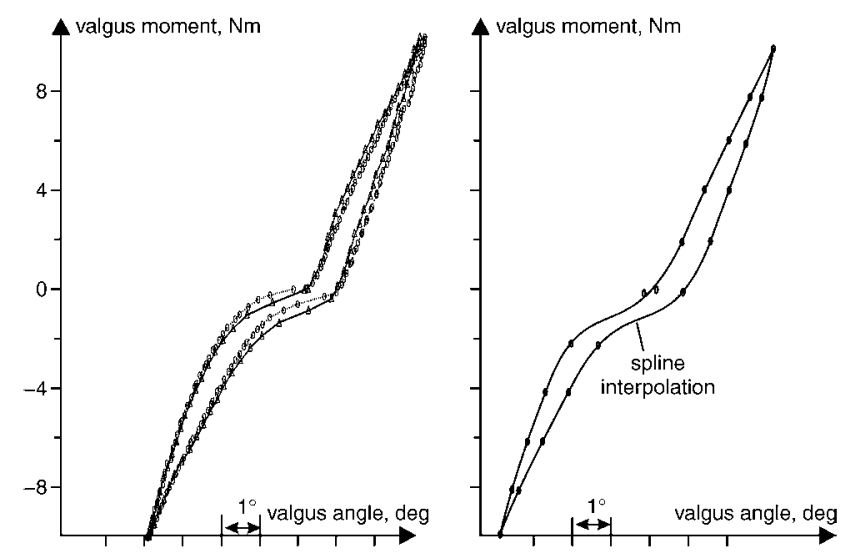

Fig. 7 Measurement of varus-valgus stiffness. Flexion angle $80^{\circ}$

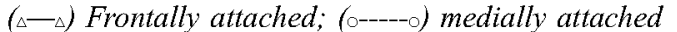

First, the novel control hardware of the robot should bring benefits from all the advantages associated with the use of high-precision industrial robots. High position accuracy, a high degree of stiffness and the ability to move the EE in 6 DOF are applicable at a sampling rate increased by a factor of $50-100$ up to $4 \mathrm{kHz}$.

Secondly, previous robot based set-ups have used sequential control strategies (FUIJE et al., 1993; RUDY et al., 1996). Within these strategies, the desired position is calculated by an outer loop and processed to a closed-loop position controller. If the desired position is reached, the next desired position is calculated. Between two steps of the outer loop, it is not possible to interfere with the movement of the robot. Furthermore, the sampling rate of the outer loop is well below $100 \mathrm{~Hz}$. This causes stability problems during force control (LAURENCE, 1988; COLGATE and BROWN, 1994).

In contrast, the set-up presented uses a quasi-continuous closed-loop controller that has a time delay that is only $0.25 \mathrm{~ms}$. Thus it behaves like a continuous controller that reacts to the applied force without appreciable delay. Because of the high speed of the new controller, it is possible to perform numerous measurements with a single cadaver and with high spatial resolution, in spite of the limited time due to the disaggregation of the cadaver. Furthermore, high spatial resolution facilitates the recognition of details, as shown in the results. Thus more accurate and even new insights into joint kinematics can be obtained when joints are analysed with high spatial resolution.

Thirdly, the sophisticated initialisation routine enables the cadaver leg to be matched with the joint CSs, which are defined in the 3D reconstructed CT data. This enables kinematics and anatomy to be aligned on-line during the measurements or afterwards, when the recorded data are analysed. Thus it is now possible to correlate changes in the anatomy, e.g. after an operation, with changes in the kinematics. This expands the capabilities to refining operative techniques and enables exact analyses of the physiological biomechanics and pathological changes.

Fourthly, the measurement protocol can be easily adapted to any application. No modification of the mechanical set-up is necessary, as the protocol is specified in the software, and both the control hardware and software are able to move the EE and to record the position and the load in $6 \mathrm{DOF}$. 
The collected data form the basis on which to generate a comprehensive dynamic biomechanical model of the intact and the ACL ruptured knee joint. The model describes the joint movement as a function of the applied load during a physical examination with clinical knee joint tests.

Thus the set-up is designed to measure elastic joint properties under load conditions that usually occur during an examination of the knee joint by an orthopaedic surgeon (FREY et al., 2003a; b). As the maximum payload of the robot is limited, it is not possible to simulate the loads that typically appear during standing and walking. However, the methods are transferable to any industrial robot with higher payload. Then, we must be aware of the fact that the accuracy decreases with increasing payload. In this case, the presented control strategy becomes even more important, as stability problems increase with lower sensor accuracy and increasing measurement noise.

The use of a 6 DOF industrial robot and of a 6 DOF FTS in combination with novel control strategies, with a routine to match anatomy and biomechanics and with modern tools for computer-based data analysis, will enhance the examination of elastic joint properties.

Acknowledgments - The author would like to thank J. Hoogen, G. Schmidt, F. Freyberger, T. Pröll and M. Buss for their assistance in this study.

This work was supported by the German Ministry for Education \& Research within the project network on 'Virtual Orthopedic Reality (VOR)', project number 01 IR A15.

\section{References}

BerNs, G. S., Hull, M. L., and Patterson, H. A. (1990): 'Implementation of a five degree of freedom automated system to determine knee flexibility in vitro', Trans. ASME, 112, pp. 392-400

Blacharski, P. A., SomerseT, J. H., and Murray, D. G. (1975): 'A three-dimensional study of the kinematics of the human knee', J. Biomech., 8, pp. 375-384

Blankevoort, L., Hutskes, R., and De Lange, A. (1988): 'The envelope of passive knee joint motions', J. Biomech., 21, pp. 705-720

Brantignan, O. C., and Voshell, A. F. (1941): 'The mechanics of the ligaments and menisci of the knee joint', J. Bone Joint Surg., 13, pp. 44-66

Colgate, J. E., and Brown, J. M. (1994): 'Factors affecting the z-width of a haptic display'. Proc. IEEE 1994 Int. Conf. Robotics \& Automation, San Diego, CA, pp. 3205-3210

Durselen, L., Claes, L., and Kiefer, H. (1995): The influence of muscle forces and external loads on cruciate ligament strain', Am.J. Sports Med., 23, pp. 129-136

Frey, M., Pröll, T., Riener, R., and Burgkart, R. (2003a): 'Making biomechanics sensible'. Proc. Workshop on Computer Simulation in Biomechanics (ISCSB), Sydney, Australia

FreY, M., PröLL, T., RIENER, R., and BURGKART, R. (2003b): 'Varusvalgus stiffness model of the knee identified by a robot based approach'. Proc. Anniversary of International Society of Biomechanics (ISB), Dunedin, New Zealand

Fujie, H., MABUChI, K., WoO, S. L.-Y., LiveSAY, G. A., ARAI, S., and TsuKAmoto, Y. (1993): 'The use of robotics technology to study human joint kinematics: a new methodology', J. Biomed. Eng., 115, pp. 211-217

Fujie, H., Livesay, G. A., WoO, S. L-Y., Kashiwaguchi, S., and Blomstrom, G. (1995): 'The use of a universal force-moment sensor to determine in-situ forces in ligaments: a new methodology', J. Biomed. Eng., 117, pp. 1-7

Fukubayashi, T., TORZILli, P. A., Sherman, M. F., and Warren, R. F. (1982): 'An in vitro biomechanical evaluation of anterior-posterior motion of the knee', J. Bone Joint Surg., 64-A, pp. 258-264

GROOD, E. S., and SUNTAY, W. J. (1983): 'A joint coordinate system for clinical description of three-dimensional motions: application to the knee', J. Biomech. Eng., 105, pp. 136-144

Hoogen, J., Ponikvar, M., and SchmidT, G. (2002): 'A robotic haptic interface for kinaesthetic knee joint simulation'. Proc. 11th
Int. Workshop on Robotics in Alpe-Adria-Danube Region, Balatonfuret, Hungary

Hurschler, C., Wülker, N., Windhagen, H., Plumhoff, P., and HELLMERS, N. (2001): 'Medially based anterior capsular shift of the glenohumeral joint-passive range of motion and posterior capsular strain', Am. J. Sports Med., 29, pp. 346-353

LAURENCE, D. A. (1988): 'Impedance control stability properties in common implementations'. Proc. IEEE Int. Conf. Robotics \& Automation, Philadelphia, Pennsylvania, pp. 1185-1190

Markolf, K. L., Mensch, J. S., and Amstutz, H. C. (1976): 'Stiffness and laxity of the knee-The contributions of the supporting structures', J. Bone Joint Surg., 58-A, pp. 583-593

MoOre, T. M., Meyers, M. H., and Harvey, J. P. (1976): 'Collateral ligament laxity of the knee. Long-term comparison between plateau fractures and normal', J. Bone Joint Surg., 58, pp. 594-598

Nemec, B., Veselko, M., RANDL, T., and AL MAWED, S. (2000): 'The use of robotics technology for kinematic test of synovial joints in medicine'. Proc. 9th Int. Workshop on Robotics in Alpe-AdriaDanube Region, Maribor, Slovenia, pp. 251-256

Piziali, R. L., and Rastegar, J. C. (1977): 'Measurement of the nonlinear, couplet stiffness characteristics of the human knee', J. Biomech., 10, pp. 45-51

RIENER, R., and EDRICH, T. (1999): 'Identification of passive elastic joint moments in the lower extremities', J. Biomech., 32, pp. 539-544

Riener, R., Frey, M., PröLl, T., REgenfelder, F., and BURgKarT, R. (2004): 'A novel mixed-reality approach for the enhancement of medical education and training', IEEE Trans. Inform. Technol. Biomed., 8, pp. 208-216

Ritchie, J., Miller, M., and HARner, C. D. (1994): 'History and physical evaluation', in FU, F. H., HARNER, C. D., and VINCE, K. G.: 'Knee surgery, Vol. 1' (Williams \& Wilkins, Baltimore, 1994)

RudY, T. W., Livesay, G. A., WoO, S. L.-Y., and FU, F. H. (1996): 'A combined robotic/universal force sensor approach to determine in situ forces of the knee ligaments', J. Biomech., 29, pp. 1357-1360

TORZILLI, P. A., GREENBERG, R. L., and INSALL, J. (1981): 'An in vivo biomechanical evaluation of anterior-posterior motion of the knee', J. Bone Joint Surg., 63-A, pp. 960-968

Woo, S. L.-Y., Debski, R. E., Vangura, A. J., Withrow, J. D., VOGRIN, T. M., WONG, E. K., and FU, F. H. (2000): 'Use of robot technology to study the biomechanics of ligaments and their replacements', Operat. Techniques Orthop., 10, pp. 87-91

WU, G., and CAVANAGH, P. R. (1995): 'ISB recommendations for standardization in the reporting of kinematic data', J. Biomech., 28 , pp. $1257-1261$

\section{Authors' biographies}

MARTIN FREY received the Dipl.-Ing. from Universität Karlsruhe (TH). After three years as a research assistant at TU Munich he became a member of the Rehabilitation Engineering Group at ETH Zurich in May 2004. His research interests are virtual reality and haptic display technologies.

RAINER BURGKART, MD had two research fellowships at Duke University/NC (1990-91 and 1998-99). Since 1997 he has been working as an Assistant Professor (Oberarzt) in Orthopaedic Surgery in the Orthopaedic Department of the Technical University of Munich. His current research interests involve innovative surgical interventions with navigation/robotics, biomechanics, simulation technology, haptic display technologies and advanced teaching devices.

FELIX REgenfeLder studied human medicine in Munich. From 2001 to 2003 he worked for the VR project as an assistant at the orthopaedic clinic of the TU Munich. He is now a resident in the surgical department in Rotenburg, Fulda.

ROBERT RIENER received the Dipl.-Ing. and the Dr.-Ing. from the TU Munich in 1993 and 1997, respectively. After postdoctoral work at the Politecnico Milan, he pursued his habilitation in Biomechatronics at TU Munich. Since 2003 he has been Assistant Professor for Rehabilitation Engineering at ETH and University Hospital Balgrist, Zurich. His research interests involve neuroprosthetics, virtual reality, and rehabilitation robotics. 